\title{
Tumor-derived exosomal components: the multifaceted roles and mechanisms in breast cancer metastasis
}

\author{
Yufang Tan (1), Xiao Luo', Wenchang Lv', Weijie Hu', Chongru Zhao', Mingchen Xiong ${ }^{1}, Y_{i}$ Yi $^{1}$, Dawei Wang ${ }^{1}$, \\ Yichen Wang ${ }^{1}$, Haiping Wang (1)', Yiping Wu $\mathbb{B}^{1}$ and Qi Zhang $\mathbb{D}^{1}$
}

\begin{abstract}
Breast cancer (BC) is the most frequently invasive malignancy and the leading cause of tumor-related mortality among women worldwide. Cancer metastasis is a complex, multistage process, which eventually causes tumor cells to colonize and grow at the metastatic site. Distant organ metastases are the major obstacles to the management of advanced BC patients. Notably, exosomes are defined as specialized membrane-enclosed extracellular vesicles with specific biomarkers, which are found in a wide variety of body fluids. Recent studies have demonstrated that exosomes are essential mediators in shaping the tumor microenvironment and BC metastasis. The transferred tumorderived exosomes modify the capability of invasive behavior and organ-specific metastasis in recipient cells. BC exosomal components, mainly including noncoding RNAs (ncRNAs), proteins, lipids, are the most investigated components in BC metastasis. In this review, we have emphasized the multifaceted roles and mechanisms of tumorderived exosomes in $\mathrm{BC}$ metastasis based on these important components. The underlying mechanisms mainly include the invasion behavior change, tumor vascularization, the disruption of the vascular barrier, and the colonization of the targeted organ. Understanding the significance of tumor-derived exosomal components in $\mathrm{BC}$ metastasis is critical for yielding novel routes of BC intervention.
\end{abstract}

\section{Facts}

Heterotypic exosome transfer in the TME supports the progression, dissemination, and metastasis of breast cancer.

Exosome-derived components, including proteins, lipids, and nucleic acids, are integral to mediate local and distant cell communication in TME.

The blocking or inhibition of exosome-derived components might represent a growing body of exciting strategies for combating metastasis.
Correspondence: Haiping Wang (Wanghpwanghp@163.com) or Yiping Wu (tongjiplastic@163.com) or Qi Zhang (zhangqi06172@163.com) 'Department of Plastic and Cosmetic Surgery, Tongji Hospital, Tongji Medical College, Huazhong University of Science and Technology, 430030 Wuhan, China These authors contributed equally: Yufang Tan, Xiao Luo, Wenchang Lv Edited by S. Tait

\section{Open questions}

How do exosomes derived from tumor act as pivotal carriers involved in tumor invasion and metastasis? Can dysregulated exosomal ncRNA and protein levels be used as potential markers of growth and metastasis for breast cancer diagnosis?

How to develop effective approaches to inhibit tumor metastasis based on exosome components?

What are the effects and mechanisms of these exosome components on the conventional treatment of breast cancer?

\section{Introduction}

Breast cancer (BC) is the most common female malignancy and one of the leading causes of cancer-related death all over the world ${ }^{1}$. As a highly heterogeneous

\section{(c) The Author(s) 2021}

(c) Open Access This article is licensed under a Creative Commons Attribution 4.0 International License, which permits use, sharing, adaptation, distribution and reproduction c. in any medium or format, as long as you give appropriate credit to the original author(s) and the source, provide a link to the Creative Commons license, and indicate if changes were made. The images or other third party material in this article are included in the article's Creative Commons license, unless indicated otherwise in a credit line to the material. If material is not included in the article's Creative Commons license and your intended use is not permitted by statutory regulation or exceeds the permitted use, you will need to obtain permission directly from the copyright holder. To view a copy of this license, visit http://creativecommons.org/licenses/by/4.0/. 


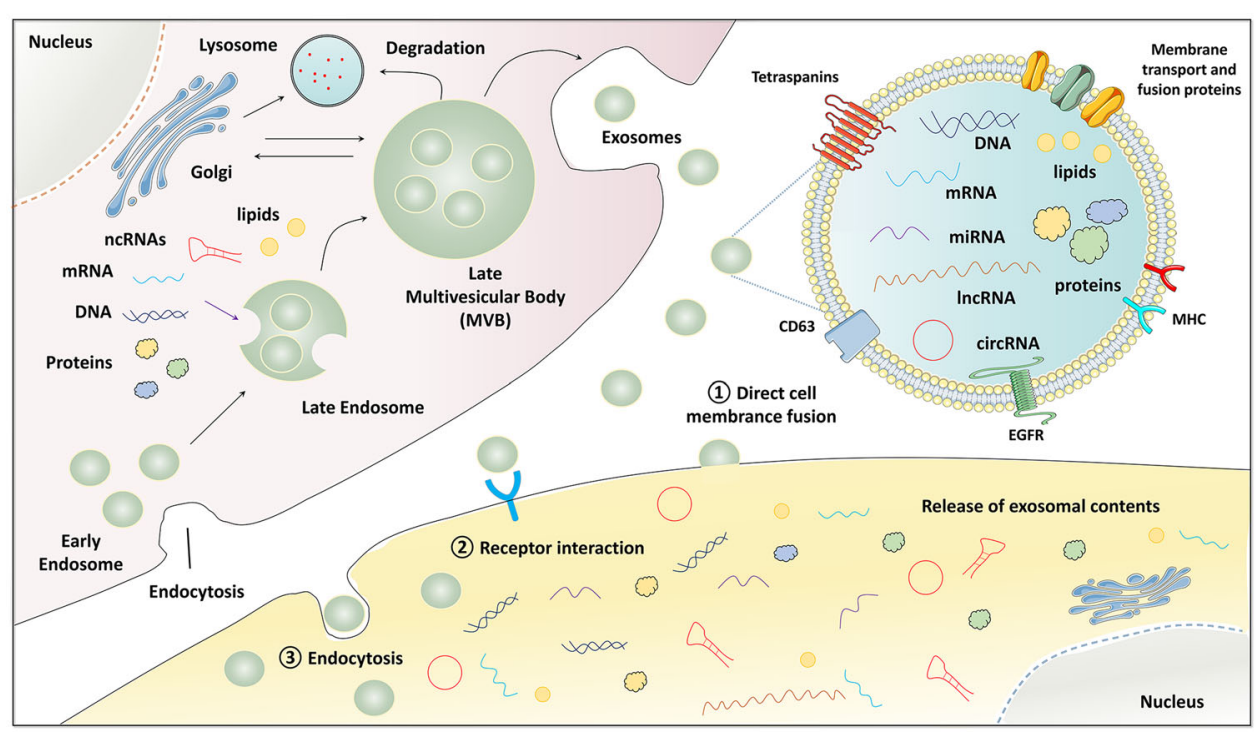

Fig. 1 The biogenesis, secretion, and uptake of tumor-derived exosomes. Firstly, the endocytosis results in the formation of early endosomes. Then, the endosomes and selected cargos including nucleic acids, proteins, and lipids are encapsulated into multivesicular bodies (MVBs). Finally, these MVBs fuse with the plasma membrane and release exosomes into extracellular place. The release of exosomal contents (proteins, mRNAs, miRNAs, IncRNAs, circRNAs, and DNAs) to the recipient cells via direct cell membrane fusion, receptor interaction, and endocytosis. noncoding RNA ncRNA, microRNA miRNA, long noncoding RNA IncRNA, circular RNA circRNA, major histocompatibility complex MHC, epidermal growth factor receptor EGFR, late multivesicular body MVB.

disease, $\mathrm{BC}$ can be classified into various subtypes including luminal A, luminal B, HER2-enriched, basallike, and normal-like according to the genetic and clinical features ${ }^{2}$. Despite the progress of early detections and relatively effective therapies such as mastectomy, radiotherapy, and chemotherapy, $\mathrm{BC}$ is still being confronted with high invasion, metastasis, recurrence rate, and drug resistance ${ }^{3}$. Treatment failure and poor prognosis caused by distant organ metastases are major obstacles to the management of advanced $\mathrm{BC}$ patients. Therefore, there is an urgent need to illustrate the key molecular events leading to the malignancy of metastatic breast cancer $(\mathrm{mBC})^{4}$.

Exosomes are defined as specialized membraneenclosed extracellular vesicles with a particle diameter size of $50-200 \mathrm{~nm}^{5}$. In terms of the specific biomarkers, exosomes are enriched in a group of conserved proteins, including the tetraspanin family (CD63, CD9, and CD81), the endosomal trafficking proteins (endosomal sorting complex required for transport (ESCRT)-related proteins/ Alix), and heat-shock proteins Hsp60, Hsp70, and Hsp90 ${ }^{6}$. The biogenesis and release of exosomes into the extracellular space need to undergo initiation, endocytosis, multivesicular body (MVB) formation, and exosome secretion. Since exosomes are possibly released from almost all cell types, they are widely distributed in the body fluids such as blood, urine, saliva, amniotic fluid, and breast milk, under both healthy and pathological conditions ${ }^{7}$. Importantly, exosomes can deliver multiple functional cargos into the extracellular space, including proteins, lipids, noncoding RNAs (ncRNAs), mRNAs, and DNA fragments ${ }^{8}$. These exosome components act as mediators of cell-to-cell communications locally and systemically by inducing phenotypic alterations in recipient cells (Fig. 1). Therefore, exosomes can impact neighboring or distant cells to modulate antigen presentation, immune function, angiogenesis, cell proliferation, tumor cell migration, and invasion'.

Metastasis of $\mathrm{BC}$ is a multistep evolutionary process by which the cancer cells transcend their programmed behavior to disseminate from the original sites, intravasate into the lymphatic system and blood circulation, and eventually extravasate to colonize distant $\operatorname{sites}^{10}$ (Fig. 2). Increasing evidence has shown that exosomes play an irreplaceable role in regulating the $\mathrm{BC}$ process and aggressive phenotype ${ }^{11}$. Tumor-derived exosomes have been shown to regulate pre-metastatic niche formation, organotropism, migration, invasion, stemness, and survival. For instance, $\mathrm{BC}$-derived exosomes could promote the ability of BC-cell proliferation, motility, and metastasis, thus generating an enhanced oncogenic phenotype ${ }^{12}$. In addition, Piao et al. reported that $\mathrm{BC}$ cell-derived exosomes stimulated macrophage polarization that established favorable conditions for metastatic processes of the lymph node in triple-negative breast cancer $(\mathrm{TNBC})^{13}$. Mechanismly, tumor-derived exosomes are able to condition the pre-metastatic niche microenvironment by manipulating the metastatic cascade through 


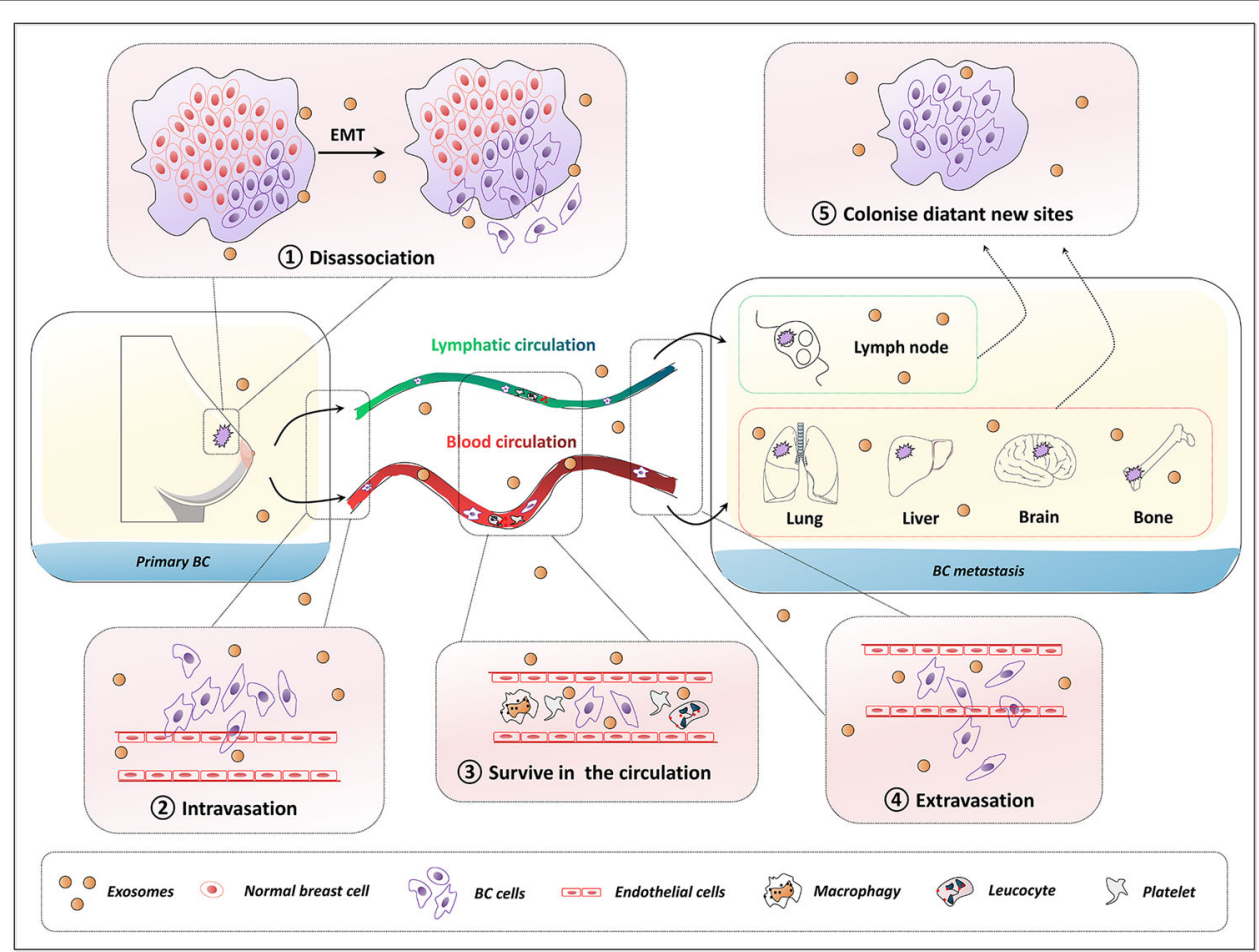

Fig. 2 The general multistep process of BC metastasis and exosomes act as regulatory factors. Metastasis is a multistep process by which the primary BC cells undergo epithelial-mesenchymal transition (EMT) and dissociate from the initial site, intravasation, survival in the blood or lymphatic circulation, extravasation, and ultimately colonize distant sites. Exosomes from BC cells are crucial orchestrators to condition the pre-metastatic niche microenvironment in metastatic progression. Breast cancer BC, epithelial-mesenchymal transition EMT.

angiogenesis, immune reaction modulation, signal transduction, and genetic intercellular exchange ${ }^{14}$.

The ncRNAs, mainly including microRNA (miRNA), long noncoding RNA (lncRNA), circular RNAs (circRNAs), are defined as genes that are transcribed into RNAs, but not translated into proteins, accompanied with the ability to regulate gene expression at the levels of transcription, RNA processing, and translation ${ }^{15}$. Considering that exosomes are key carriers of juxtacrine and paracrine signaling for mediating metastatic progression, the large number and the versatility of ncRNAs and proteins in exosomes have fueled many studies on their roles in BC metastasis. Herein, we have summarized the multifaceted roles and mechanisms of tumor-derived exosomes in $\mathrm{BC}$ metastasis based on these important components. It will help decipher how the tumor-derived exosomes reshape the tumor microenvironment (TME) and the $\mathrm{BC}$ metastasis and will provide novel and effective therapeutic strategies for combating $\mathrm{mBC}$.

\section{Factors promoting tumor-derived exosome biogenesis, release, and uptake for BC metastasis}

Cancer metastasis is a complex, multistage process, which eventually causes tumor cells to colonize and grow at the metastatic site ${ }^{16}$. Exosomes derived from tumors and stromal cells have been involved in all stages of cancer metastasis. In addition to the different types of substances loaded in exosomes, the content of tumor-derived exosomes also impacts $\mathrm{BC}$ metastasis. Understanding the cellular basis of exosome biogenesis, release and uptake is critically important for developing targeted therapies by blocking exosomes.

The biogenesis of exosomes is a complex multistep process. Firstly, the endocytosis in lipid raft domains of the plasma membrane results in the intracellular formation of early endosomes. Then, under the participation of the Golgi apparatus, the interluminal vesicles (ILV) buds from endosomal compartments and further connect together, forming the MVB, which means that early endosomes transform into late endosomes accordingly. This conversion is accompanied by a cargo sorting procedure for the recruitment of molecular contents into ILVs, including proteins, nuclear acids, and metabolites. It is generally acknowledged that the mechanism of the exosomal formation processes mainly includes two ways: the ESCRT pathway and the ESCRT-independent pathway. The ESCRT pathway is a complex of four proteins (ESCRT-0-III) including Hrs, CHMP4, TSG101, STAM1, 
VPS4 that promote MVB formation, budding, and cargo distribution ${ }^{17}$. Lipid raft microdomains play a crucial role in the MVB biogenesis in an ESCRT-independent manner ${ }^{18}$. Meanwhile, a family of 4-transmembrane proteins (tetraspanins), such as CD9, CD63, CD81, CD82, and CD97, are also linked to the formation of ESCRTindependent exosomes ${ }^{19}$. These are all key factors in exosome genesis and regulation, indicating the complexity between cell function and exosome activity/quantity.

Finally, some MVBs bind to lysosomes for degradation, while the other MVBs are guided to the cell membrane, anchor, and fuse with the cell membrane, thus releasing internal ILVs to the outside of the cell to form exosomes. The exocytosis of the ILV-containing MVBs is mainly driven by Rab GTPases at the plasma membrane, specifically RAB27A and RAB27B. Ghoroghi et al. identified RalA/B GTPases were a novel molecular mechanism that could control exosome secretion levels via the homeostasis regulation of $\mathrm{MVBs}^{20}$. The RalA/B-phospholipase D1 (PLD1)-phosphatidic acid (PA) axis governed exosome biogenesis to a certain extent. RalA and RalB reduced the EV levels of the adhesion molecule MCAM/ CD146, which was in favor to disseminate and induce the formation of pre-metastatic niches in a CD146-dependent manner. Additionally, SNARE protein complex, syntaxin $1 \mathrm{~A}$, synaptotagmin protein family, $\mathrm{Wnt}$, and $\mathrm{Ca} 2+$ also participate in regulating the process of release of exosomes $^{19}$. Interestingly, Pan et al. found alter levels of $\mathrm{Ca} 2$ + affected exosome release from MDA-MB-231 cells, and therefore exerted an impact on angiogenesis of human umbilical vein endothelial cells (HUVECs) ${ }^{21}$. Hypoxic tumors exhibit more aggressive phenotypes and are associated with poor patient outcomes in a wide variety of cancers $^{22}$. Hypoxia is an important feature of solid tumors that promotes tumor progression, angiogenesis, and metastasis, potentially through exosome-mediated signaling ${ }^{23}$. King et al. proved that hypoxia promoted the release of exosomes by $\mathrm{BC}$ cells to promote survival and invasion, and that this hypoxic response might be mediated by hypoxia-inducible factor- $1 \alpha(\mathrm{HIF}-1 \alpha)^{24}$. Furthermore, the hypoxically regulated miR-210 was identified to be present at elevated levels in hypoxic exosome fractions. The study of Wang et al. suggested that the HIFdependent RAB22A gene expression level was upregulated by hypoxia in advanced $\mathrm{BC}$, which promoted microvesicle generation and stimulated $\mathrm{BC}$ invasion ${ }^{25}$. In view of the emerging role of tumor cell-derived exosomes in tumor progression, it is of great significance for understanding the inner link of hypoxic and exosome biogenesis.

Asparaginyl $\beta$-hydroxylase (ASPH) gene is upregulated and is required for the generation and maintenance of malignant phenotypes in invasive $\mathrm{BC}^{26}$. Lin et al. showed ASPH-Notch axis resulted in matrix metalloproteinases
(MMPs)/ADAMs mediated extracellular matrix (ECM) degradation/remodeling, benefiting invasiveness by particularly guiding exosomal delivery of pro-metastatic secretome ${ }^{27}$. The crucial role of ASPH in promoting invasion, aggressiveness, and multi-organ dissemination, making it a potential therapeutic target for $\mathrm{BC}$ metastasis. Autophagy and autophagy-related genes (Atg) also occupy prominent roles in oncogenesis, growth, and metastasis of $\mathrm{BC}^{28}$. The Atg5 disassociated the $\mathrm{V}_{1} \mathrm{~V}_{0^{-}}$ ATPase to promote exosome production and tumor metastasis in vivo independent of canonical macroautophagy ${ }^{29}$. Endothelin receptor A (ETA), belonging to a member of the $\mathrm{G}$ protein-coupled receptor family, is related to various effects of $\mathrm{BC}$ growth, metastasis, and angiogenesis. ETA was important to interference with $\mathrm{BC}$ cells to promote exosome secretion in BC cells. The inhibition of ETA function by antibiotic sulfisoxazole could result in an anti-metastasis effect in mouse models of $\mathrm{BC}$ xenografts via suppressing the biogenesis and secretion of exosomes in an ETA dependent way ${ }^{30}$. Whole-genome sequencing data from human tumors found that Munc13-4, a Rab-binding protein, was elevated in BC. Munc13-4 and Ca2+ uptake increased in BC cells after epithelial-mesenchymal transition (EMT), and further promoted exosome release from highly invasive $\mathrm{BC}$ cells, inducing metastasis ultimately ${ }^{31}$. It was worth noting that Munc13-4 regulated MVB maturation and generated MVBs competent for exosome release by a Rab11dependent trafficking pathway. MAP17 was a commonly upregulated protein in inflammation and carcinomas. Furthermore, MAP17 increased the secretion of BC cellderived exosomes, and also could be released as exosomal cargoes $^{32}$. Thus, this horizontal propagation also increased the EMT in the recipient cells. MAP17 could be considered as a novel target for $\mathrm{mBC}$ attributing to its ability to shape tumor evolution.

After release, exosomes can orchestrate on their parental cells, adjacent and distant cells in an autocrine, a juxtacrine and a paracrine manner. When they reached their target cells, the first way of uptake is to recognize receptors on the surface of the cell membrane and activate the corresponding signaling pathways. The second crucial uptake mechanisms of exosomes is endocytosis, such as pinocytosis, phagocytosis and micropinocytosis. Besides, the special lipid raft structure caveolae-mediated endocytosis is also involved in the uptakes of exosome. The third way is to fusion directly with the plasma membrane of the target cells, thus releasing their cargo. It is worth mentioning that uptake quantity of exosome is also important for exosome-mediated tumor progression. For example, Esposito et al. screened the novel RNA aptamer ex-50.T, which specifically recognized exosomes derived from $\mathrm{BC}$ cells and inhibited their cellular uptake as well as interrupted exosome-mediated cell invasion, 
putting forward the possibility of tumor behavior inhibition by reducing exosomal exchange ${ }^{33}$. Besides, several commonly used agents in clinic are postulated to block exosome uptake through various cellular mechanisms, including anticoagulant heparin, antimalarial drug chloroquine, the diuretic amiloride, and antipsychotic drug chlorpromazine ${ }^{34}$.

In addition to the above extrinsic biological factors, it is important that exosome biogenesis and uptake are also influenced by several external factors, such as cell type, cell state, hypoxia, serum conditions, the added cytokines and growth factors and even the separation and purification method of exosomes ${ }^{35}$. Targeting the biogenesis, release and uptake of exosomes might be a potential therapeutic approach to change the course of cancer.

\section{Tumor-derived exosomal components in BC metastasis}

Tumor-derived exosomes deliver into the bloodstream and mediate nonrandom patterns of metastasis ${ }^{36}$. BC metastasis usually develops in multiple organs including lymph nodes, bone, lung, brain, and liver. The selective preference of tumor metastasis to certain tissues is complicated and dependent on several factors including vascular patterns, adhesion factors, the interaction of tumor cells with the matrix at the site of metastasis. It is recognized that organ-specific metastatic potential requires close interaction between cancer cells and TME. Tumor-derived exosomes have been reported to deliver ncRNAs, proteins, and lipids to incorporate into surrounding cells or cells in distant metastatic niches by conditioning pre-metastatic TME.

\section{Exosomal noncoding RNA \\ Exosomal miRNA}

The miRNAs are small ncRNAs with 19-25 nucleotides length and can regulate gene expression posttranscriptionally by binding to the complementary sites in the $3^{\prime}$ untranslated region (UTR) of targeted mRNAs ${ }^{37}$. Tumor-derived exosomes, especially carrying miRNAs, can be diffused to neighboring cells or systemically transported to the distant organ to induce malignant transformation and field cancerization ${ }^{38}$. Interestingly, in addition to carrying miRNA, serum exosomes from BC patients contained dicer and could process pre-miRNAs to generate mature miRNAs ${ }^{39}$. BC exosomes induced transcriptome alterations in recipient cells and tumor formation in a dicer-dependent manner ${ }^{39}$. Totally, exosomes play pleiotropic roles in cancer progression and metastasis, including invasion, angiogenesis, and immune modulation.

Exosomal miRNAs regulate the malignant phenotype of BC cells At early-stage $\mathrm{mBC}$, tumor cells disseminate through both lymphatic and hematogenous systems. Some miRNAs act as tumor suppressors that migrate through the vasculature by the exosome. Kong et al. found that miR-130a-3p was aberrantly downregulated in human BC tissues and exosomes from circulating blood, while the lower levels of exosome-derived miR-130a-3p were related to lymph node metastasis and advanced TNM stage ${ }^{40}$. In vitro, exosomal miR-130a-3p inhibited the cell proliferation, migration, and invasion of human breast cancer stem cells (BCSCs) by directly regulating RAB5B/epidermal growth factor receptor signaling pathways. $\mathrm{Li}$ et al. pointed out that miR-770 encapsulated in tumor exosome could be transported into tumorassociated macrophages, and thereby increased the miR770 expression in macrophages ${ }^{41}$. Additionally, miR-770 overexpression inhibited the migration and invasion of TNBC via targeting of STMN1. Let-7a and c-Myc were negatively correlated with $\mathrm{BC}$. The Let-7a derived from MDA-MB-231 cell exosomes could inhibit the proliferation, migration, and invasion both in vitro and in vivo by silencing the c-Myc expression ${ }^{42}$. It was also found that miR-188-5p inhibited BC-cell proliferation and migration, which was mediated by the downstream target interleukin 6 signal transducer (IL6ST) ${ }^{43}$. This result revealed that miR-188-5p was selectively sorted into exosomes from malignant $\mathrm{BC}$ cells, but not into patient serum exosomes. The transfer of miR-155 from BC exosomes could reprogram the metabolism in adipocytes and muscle cells to induce lipolysis and muscle loss, thus leading to cancerassociated cachexia to promote metastasis in $\mathrm{BC}^{44}$.

The exosomal miRNA secreted by $\mathrm{BC}$ cells can also dynamically promote the malignant phenotype of tumors, which is related to the refractory and progression of tumors. Ding et al. showed that exosomal miR-222 was highly upregulated and correlated to $\mathrm{BC}$ patients with lymphatic metastasis ${ }^{45}$. BC exosome-transferred miR-222 contributed to tumorigenicity and metastatic progression of $\mathrm{BC}$ cells both in vitro and in vivo, potentially mediated by tumor suppressor gene PDZ and LIM domaincontaining protein 2 (PDLIM2) downregulation and consequent NF-kB activation. Yang et al. showed that miR-146a derived from $\mathrm{BC}$ exosomes enhanced the transformation of normal fibroblasts (NFs) into cancerassociated fibroblasts (CAFs) via miR-146a/thioredoxininteracting protein (TXNIP) axis to promote the Wnt pathway in TME, contributing to the accelerated invasion and metastasis of $\mathrm{BC}$ cells ${ }^{46}$. Wang et al. confirmed the exosomal miR-1910-3p enhanced the tumorigenicity in the tumor xenograft model ${ }^{47}$. The miR-1910-3p was enriched in BC-cell exosomes and could transfer to mammary epithelial cells and BC cells, ulteriorly to promote growth, metastasis, and autophagy of $\mathrm{BC}$ cells by regulating myotubularin-related protein 3 (MTMR3) and activating the NF- $\mathrm{KB}$ signaling pathway. The 
expression levels of miR-200c and miR-141 were higher in plasma from patients with $\mathrm{mBC}$ than localized $\mathrm{BC}$ or healthy controls, possessing the potential to be biomarkers for early detection of tumor metastasis ${ }^{48}$. These circulating miR-200c and miR-141 probably were secreted or released from circulating tumor cells mediated by exosomes. Moreover, miR-200c and miR-141 were reregulated by a FOXP3-KAT2B axis in BC cells.

MiR-155 is a well-known oncogenic miRNA, which is often upregulated in $\mathrm{BC}$, and is widely involved in the recurrence, metastasis, and treatment resistance of $\mathrm{BC}$. miR-155 was enriched and could be separated from cancer stem cells (CSCs) and resistant cells, accompanied by the transfer of miR-155 exosomes to $\mathrm{BC}$ cells ${ }^{49}$. Exosomes remodeled the migration capacity with enhanced EMT to sensitive cells partly by exosomal transfer of miR-155. The research of Gorczynski et al. confirmed that both miR-155 and miR-205 could potentiate the inflammatory responses to modulate the metastatic growth of $\mathrm{BC}$ cells in the lung and liver metastasis model. Increased BC exosomal miR-205 was reported to attenuate BC metastasis while miR-155 had the opposite effect ${ }^{50}$. By bioinformatic approach, Kia et al. found that miR-9 and miR-155 were among the overexpressed miRNAs in highly-metastatic TNBC cells and their exosomes, which was further validated by qRT$\mathrm{PCR}^{51}$. Luciferase assay verified that the $\mathrm{BC}$ exosomal miR-9 and miR-155 targeted the UTRs of tumor suppressors phosphatase and tensin homolog (PTEN) and dual-specificity phosphatases 14 (DUSP14), respectively. Interestingly, in their following study, the metastatic behavior of low-metastatic recipient MCF-7 cells with the treatment of highly-metastatic MDA-MB-231 cells exosomes acquired enhanced metastatic phenotype $^{52}$. The miR-155 shuttled by exosomes introduced a novel behavior with the increased ability of cancer development and metastasis. The elevated miR-7641 in $\mathrm{BC}$-derived exosomes was found in the plasma of $\mathrm{BC}$ patients with distant metastasis and could promote tumor growth both in vitro and in vivo ${ }^{53}$. MiR-7641 could promote $\mathrm{BC}$-cell proliferation and invasion, and induce epigenetic modulation in recipient cells via exosome transfer.

Exosomal miRNAs induce angiogenesis of tumor Pathological angiogenesis could provide nutrients and oxygen for tumors, thus facilitating the growth and dissemination of cancers and distant metastasis. Exosomes are crucial mediators in their regulation of angiogenesis and cell migration in tumor growth and spread. MiRNAs are emerging regulatory RNAs that can be selectively encapsulated into exosomes and function as a messenger in intercellular communication to regulate tumor metastasis and angiogenesis, and remodel the
TME. Feng et al. showed that BC cells secreted exosomal miR-22-3p mediated tumor vessel abnormalization by suppressing transgelin, thus promoting tumor budding and $\mathrm{BC}$ progression in vivo ${ }^{54}$. Pan et al. found exosomal miR-145 in MDA-MB-231 cells targeted IRS1 and inhibited the angiogenesis of HUVECs via regulating IRS1/PI3K/Akt/mTOR and IRS1/Raf/ERK pathways ${ }^{21}$. Furthermore, stromal interaction molecule 1 (STIM1), which was a transmembrane protein located in the endoplasmic reticulum, reduced BC exosomal miR-145 to promote angiogenesis and migration.

\section{Exosomal miRNAs mediate disruption of vascular} barrier In the process of metastatic and diffusion, $\mathrm{BC}$ gains the ability to transmigrate through blood vessels by inducing alterations in the endothelial barrier. As reported by Modica et al. the exosomal miR-939 in TNBC cells increased tumor cell trans-endothelial migration and directly targeted vascular endothelial cadherin (VE-cadherin) in endothelial cells ${ }^{55}$. This work suggested that $\mathrm{BC}$-secreted exosomal miR-939 was involved in the extracellular pro-tumorigenic characteristic and was associated with a worse prognosis in TNBCs. miR-105 was characteristically secreted by $\mathrm{mBC}$ cells and was a potent migration regulator by targeting the tight junction protein zona occluden-1 (ZO- 1$)^{56}$. In endothelial monolayers, the BC-secreted exosomal miR-105 effectually damaged the integrity of natural barriers and benefited for metastasis. Clinically, miR-105 could be detected in the circulation at the pre-metastatic stage, and its levels in the blood and tumor were correlated with ZO-1 expression and metastatic progression in early-stage $\mathrm{BC}$. RodríguezMartínez et al. Before neoadjuvant therapy, exosomal miRNA-21, and 105 expression levels were higher in metastatic versus nonmetastatic patients and healthy donors ${ }^{57}$.

Exosomal miRNAs mediate colonization of targeted organ In the specific organ metastasis, tumor-derived exosomal miRNAs not only show aberrant expression in content, but also exhibit the ability to prepare the premetastatic niche with specific organ-oriented invasion ability. miR-20a-5p was overexpressed in BC tissues and the exosomes of MDA-MB-231 cells ${ }^{58}$. This exosomal miR-20a-5p promoted the migration and invasion in MDA-MB-231 cells, and could be transferred to bone marrow macrophages (BMMs) and facilitated the osteoclastogenesis via targeting SRC kinase signaling inhibitor 1 (SRCIN1). BC cell-secreted exosomes might be a prerequisite for pre-metastatic niche generation by promoting osteoclast differentiation and enhancing bone metastasis. Yuan et al. provided evidence that $\mathrm{BC}$ patients with bone metastasis showed higher expression of exosomal miR-21 in serum than those without metastasis 
or with non-bone tissue metastasis ${ }^{59}$. In experimental exploration, exosomal miR-21 derived from highmetastatic SCP28 cells boosted osteoclastogenesis via regulating programmed cell death 4 (PDCD4) protein levels, resulting in the formation of the premetastatic niche.

In the lung metastatic mouse model, the $\mathrm{BC}$ exosomal miR-183-5p could transfer to macrophages to promote the secretion of pro-inflammatory cytokines interleukin$1 \mathrm{~b}$ (IL-1 $\beta$ ), IL-6, and tumor necrosis factor- $\alpha$, via repressing phosphatase 2 catalytic subunit alpha $(\mathrm{PPP} 2 \mathrm{CA})^{60}$. Obviously, the knockdown of miR-183-5p in 4T1 exosomes inhibited $\mathrm{BC}$ growth and metastasis in the $\mathrm{BC}$ lung metastasis model. In another search, mimic miR-33 delivered by $4 \mathrm{~T} 1$ cell exosome was able to induce M1 polarization in macrophages, resulted in improved antitumor effect ${ }^{61}$. It was known that tissue inhibitors of metalloproteinase 2 (TIMP2) belonged to the TIMP family and could effectively inhibit the invasion and metastasis of tumors. Wang et al. confirmed that in the BC metastatic mouse model, the overexpression of tumor-derived miR-4443 was of high capacity to induce $\mathrm{BC}$ liver metastasis ${ }^{38}$. The conveying exosomal miR-4443 could break the natural barriers, which was accompanied by impaired TIMP2 and consequently upregulated MMP2 in both the primary tumor and metastasis liver sites, resulted in engrafting in the new microenvironment.

The metabolic profile of most cancer cells resulted in increased aerobic glycolysis with lowered mitochondrial oxidative phosphorylation in an aerobic environment, which is known as the "Warburg effect" to favor the uptake and incorporation of nutrients. By secreting vesicles including exosomes with high levels of the miR122 , BC cells suppressed the glucose uptake of non-tumor cells in the pre-metastatic niche, by downregulating the glycolytic enzyme pyruvate kinase ${ }^{62}$. Intriguingly, the in vivo inhibition of miR-122 significantly restored the efficacy of glucose uptake in distant organs, including the brain and lung, and thus reduced the metastasis incidence. That meant, BC-secreted miR-122 reprogrammed glucose consumption in niche tissues and promoted metastasis. By profiling lncRNAs, Xing et al. found that X-inactive-specific transcript (XIST) was significantly downregulated in tumors with breast cancer brain metastasis $(\mathrm{BCBM})^{63}$. Loss of XIST in BC activated MSN-c-Met and reprogrammed microglia via exosomal miRNA-503, which triggered M1-M2 polarization of microglia and suppressed T-cell proliferation to promote brain metastasis. Sharma et al. utilized a novel therapy for BCBM by delivering athermal radiofrequency electromagnetic fields that are amplitude-modulated at breast cancer-specific frequencies $(\mathrm{BCF})^{64}$. It was interesting that BCF treatment could inhibit the growth of brain metastasis in a mouse model. The result showed that
BCF suppressed angiogenesis in the TME by inhibiting $\beta$-catenin and decreasing the exosomal secretion of miR1246. Coincidently, the research of Li et al. also confirmed that exosomal miR-1246 was highly expressed in $\mathrm{mBC}$ MDA-MB-231 cells compared to nonmetastatic or nonmalignant $\mathrm{BC}$ cells ${ }^{65}$. Besides, exosomal miR-1246 boosted cell proliferation and invasion by inhibiting the expression level of cyclin-G2.

Based on the above studies, it is not hard to see that tumor-derived exosomal miRNAs transfer functions or characteristics into recipient cells. These abundant miRNAs are the key mediators for the bidirectional communication of tumor cells and other cell types, involving all the metastasis processes including the tumor cell dissemination from the primary tumor site, TME formation, and tumor cell colonization in a distant organ. These miRNAs are potential candidates for constituting unique molecular features, identifying clinically primary subtypes, and being therapeutic targets of $\mathrm{BC}$.

\section{Exosomal IncRNA}

LncRNAs are a category of cellular ncRNAs with a length of more than 200 nucleotides. Recently, lncRNAs have become critical components in cancer evolution by playing a driving role in suppressing and carcinogenic functions in prevalent cancer types, such as $B^{66}$. For example, a lncRNA associated with BCBM (Lnc-BM) is a prognostic indicator of brain metastasis progression in patients with $\mathrm{BCBM}^{67}$. Lnc-BM increased Janus kinase-2 (JAK2) kinase activity to trigger STAT 3 phosphorylation, followed by macrophage recruitment and activation, thus promoting BCBMs by mediating communication between $\mathrm{BC}$ cells and the brain microenvironment. $\mathrm{BC}$ cell-derived exosomal small nucleolar RNA host gene 16 (SNHG16) promoted the activation of the TGF- $\beta 1 /$ SMAD 5 pathway by sponging miR-16-5p and resulted in the conversion of $\gamma \delta 1 \mathrm{~T}$ cells into the CD73+ immunosuppressive subtype $^{68}$. The lncRNA shuttled by exosome modifies the malignant behavior of the tumor showing the potential to be promising biomarkers for the diagnosis and prognosis of $\mathrm{mBC}$.

By using high-throughput sequencing analysis, Feng et al. manifested that a lot of functional involvement of abnormally expressed lncRNAs derived from BC cells induced proliferation and migration of lung fibroblasts, which provided an appropriate environment for the formation of pre-metastatic niche and facilitated tumor pulmonary metastasis ${ }^{69}$. Among them, there were a total of 64 lncRNAs co-increased expressions and 8 lncRNAs co-decreased. Liang et al. identified that hypoxiaresponsive lncRNA BCRT1 was upregulated in BC tissues, and the higher expression of lncRNA BCRT1 was associated with aggressive tumor metastasis and poor 
prognosis $^{70}$. The transmission of lncRNA BCRT1 from $\mathrm{BC}$-derived exosomes could promote $\mathrm{M} 2$ polarization and thus enhance M2 tumor-promoting function. LncRNA BCRT1 acted as a sponge for miR-1303 to weaken its silence effect on polypyrimidine tract binding protein 3 (PTBP3) expression. Lu et al. found that lncRNAGS1600G8.5 was overexpressed in exosomes derived from BC cells which easily metastasized to the brain $^{71}$. More importantly, by transferring IncRNA GS1- 600G8.5, the exosomes from the high brain $\mathrm{mBC}$ cells could be internalized by brain microvascular endothelial cells, which disrupted the permeability of the blood-brain barrier (BBB) and promoted the passage of cancer cells across the $\mathrm{BBB}$ in vivo. The in vitro assay further confirmed that the exosomal GS1-600G8.5 could destroy the BBB by inhibiting tight junction proteins.

\section{Exosomal circRNA}

circRNAs are one of the newly discovered ncRNAs, which are connected end-to-end to form covalently closed single-chain circular molecule. As highly conserved RNAs, many circRNAs contain abundant miRNA-binding sites, suggesting that they may function as miRNA sponges. Exosomal circRNAs derived from BC cells mostly act as competing endogenous RNAs (ceRNAs) in recipient cells, as they modulate miRNA-target gene expression and contribute to oncogenesis and development.

Through microarray and RNA sequence techniques, Wang et al. verified a total 1061 of upregulated circRNA and 86 of downregulated circRNA in the exosomes of patients with $\mathrm{mBC}^{72}$. Additionally, 5 circRNAs were confirmed to be elevated in $\mathrm{mBC}$ patient-derived exosomes by RT-qPCR, and possible circRNA/miRNA interactions and conceivable functional meshwork were proposed using bioinformatics methods. CircRNAs might have crosstalk with more than one miRNA and be associated with several pathways involving in BC migration and invasion. Similarly, Yang et al. verified two significantly differentially expressed circRNAs in exosomes from $\mathrm{BC}$ tissues in comparison with nontumor tissues, where hsa-circRNA-00005795 was downregulated and hsa-circRNA-0088088 was upregulated $^{73}$. Of note, regarding the role of circRNAs as miRNA sponges, a multifunctional network was structured between hsa-circRNA-00005795 and eight miRNAs and between hsa-circRNA-0088088 and 11 miRNAs respectively using three databases. These circRNAs-associated miRNAs were related to signaling pathways in the $\mathrm{BC}$ progression, including but not limited to Wnt, estrogen, and TGF- $\beta$ pathways. Ding et al. verified that circ_0004771 was higher in serum exosomes from $\mathrm{BC}$ patients in comparison to healthy patients, and could harbor miR-1253 in BC to upregulate dimethylarginine dimethylaminohydrolase 1 (DDAH1), contributing to the alteration of $\mathrm{BC}$ malignant phenotypes ${ }^{74}$. The identity, dysregulation, and function of exosomal circRNAs in BC metastasis are only beginning to be understood, thus further mechanisms are needed to ascertain the intricate roles of circRNAs in $\mathrm{mBC}$.

\section{Exosomal protein}

The proteome profile of exosomes isolated from $\mathrm{BC}$ cells contributes to different metastatic potential. Exosomes secreted by $\mathrm{mBC}$ cells are enriched in many membrane proteins. As reported by Gangoda et al. that, by proteomic profiling of highly-metastatic $4 \mathrm{~T} 1$ and nonmetastatic 67NR exosomes, the former exosomes were enriched with membrane proteins including $\mathrm{Cd} 38$, Slco2a1, Acsl4, Mtdh, Fgfr, and Tgfbr ${ }^{75}$. It could be inferred that the exosomes contained various protein cargo depending on the host cells metastatic properties and facilitated in the dissemination of the primary tumors to distant sites. The functions of exosomal proteins of $\mathrm{BC}$ cells depend on the following mechanisms, including promoting the invasion behavior, responding to the hypoxic microenvironment, inducing tumor vascularization, disrupting vascular barrier, and mediating targeted organ colonization.

\section{Exosomal proteins promote the invasion behavior of $B C$ cells}

Fibronectin (FN) is considered to be a key player to promote the $\mathrm{BC}$-cell behaviors including cell adhesion, metastasis, and carcinogenic transformation such as $\mathrm{EMT}^{76}$. $\mathrm{BC}$-derived exosomal FN was able to facilitate $\mathrm{BC}$ metastasis in vivo, with activated focal adhesion kinase/ Src-dependent and enhanced production of proinflammatory cytokines and metalloproteinase 9 $(\mathrm{MMP9})^{77}$. Furthermore, the neutralization or small interfering RNA silence of tumor-exosomal FN partially reversed the tumor exosome-mediated tumor cell invasion. Didiasova et al. found that the invasion ability of MDA-MB-231 cells was enhanced when co-cultured with the exosome secreted by cells overexpressing enolase-1 $(\mathrm{ENO}-1)^{78}$. In addition to the expression level of cell surface-bound ENO-1, the amount of exosomal ENO-1 into the extracellular space was also correlated with the invasive metastatic potential of cancer cells. It indicated that exosomal ENO-1 might gather proteolytic activity on the BC-cell surface or enlarge the cytoplasmic pool of ENO-1, thereby modulating the expression of the genes related to cell proliferation, migration, and inflammation to promote tumor progression. RAB22A was a small GTPase, belonging to the RAB protein family involved in early endosomes, Golgi bodies, and late endosomes. Sun et al. identified that the oncogenic RAB22A could be regulated by miR-193b in $\mathrm{BC}$ cells ${ }^{79}$. Exosomes lacking 
RAB22A diminish exosome-mediated growth, invasion, and migration of the recipient $\mathrm{BC}$ cells.

\section{Exosomal proteins response to the hypoxic microenvironment}

Hypoxic TME is a common characteristic of solid tumors and is often connected with aggressiveness and poor outcomes ${ }^{80}$. BC cells under hypoxic conditions secrete a large amount of exosomes, and that exosomes released from hypoxic $\mathrm{BC}$ cells promote focal adhesion formation, invasion, and metastasis, thus implicating that tumor-derived exosomes are mediators of tumor metastasis ${ }^{81}$. Sethuraman et al. proved that hypoxiainduced activation of BHLHE40 contributed to promoting cell survival and lung metastasis in vivo of $\mathrm{BC}$ by modulating exosomal secretion of heparin-binding epidermal growth factor (HBEGF ${ }^{82}$. Therefore, the HIF-BHLHE40-HBEGF axis was an important signaling mechanism to promote metastasis of BC. Metastasisassociated protein 1 (MTA1) is a transcriptional coregulator and is an upregulated protein in cancer, whose expression correlates with cancer development, unfavorable prognosis, and enhanced metastatic potential. Hannafon et al. identified the existence and confirmed the abundance of MTA1 in BC exosomes ${ }^{83}$. Functionally, MTA1 knockout in BC cells resulted in changes to hypoxia, and affected estrogen signaling and tamoxifen sensitivity that could be attenuated by the addition of MTA1 exosomes.

\section{Exosomal proteins induce angiogenesis of tumor}

$\mathrm{BC}$-derived exosomes may transmit oncogenes and pro-angiogenic signals to stromal cells and thus promote stromal remodeling, tumor vascularization, and cell invasion ${ }^{84}$. Therefore, $\mathrm{BC}$-exosomes render tumor cells more aggressive and more prone to metastasize. Annexin A2 (AnxA2), a 36-kDa calcium-dependent phospholipid-binding protein, was associated with various cancer-associated plasminogen activation, actin-cytoskeletal rearrangement, cellular adhesion, and migration ${ }^{85}$. The exosomal AnxA2 was significantly higher in malignant cells than in normal and premetastatic $\mathrm{BC}$ cells ${ }^{86}$. Moreover, in vivo analysis indicated that the exosomal AnxA2-depleted exosomes decreased the metastatic sites of the brain and lung, confirming the important role of exosomal AnxA2 in creating a favorable TME for metastasis. High expression of circulating exosomal AnxA2 was also associated with tumor grade and resulted in poor survival of the $\mathrm{BC}$ patients ${ }^{87}$. This suggested that the circulating exosomal AnxA2 could predict the prognosis of $\mathrm{BC}$ patients. In vivo Matrigel plug assay further demonstrated that a high level of exosomal AnxA2 in serum was potently induced angiogenesis in $\mathrm{BC}$ patients. Therefore, $\mathrm{BC}$ exosomal AnxA2 promoted angiogenesis and organ-specific metastasis of BC. In 2020, Liu et al. verified the specific highly active deubiquitinase ubiquitin carboxyl-terminal hydrolase isozyme L1 (UCHL1) as a candidate oncoprotein in the aggressive $\mathrm{BC}$ patient serum and $\mathrm{BC}$-cell conditioned media (CM) by deubiquitinase activity profile ${ }^{88}$. Besides, UCHL1 was specifically enriched in donor cell exosomes to promote migration and extravasation in recipient cells by upregulating TGF $\beta / S M A D$ signaling pathways, which could be antagonized by UCHL1 inhibitor. This result highlighted the significance of UCHL1containing exosomes serving as novel blood markers for early diagnosis of aggressive BC.

\section{Exosomal proteins mediate disruption of vascular barrier}

The endothelial barrier strictly maintains the homeostasis balance of blood vessels and tissues, thus regulating many processes, such as angiogenesis, immune response, and tumor invasion ${ }^{89}$. Although intravasation depends on the migration ability of tumor cells themselves, the disruption of vascular barrier integrity facilitated by molecular changes is also important to promote the ability of $\mathrm{BC}$ cells to cross the endothelial cell barriers. TNBC is characterized by high levels of stromal and intratumoral tumor-infiltrating lymphocytes (TILs), which is a potential predictive biomarker of more favorable survival outcomes and response to chemotherapy for immunotherapy response in TNBC. The crosstalk between lymphocytes and tumor cells was crucial for the modulation of the secretion in the exosomal mRNA profile of recipients. Theodoro et al. conducted an in vitro assay that the lymphocytes collected from healthy women were co-cultured with $\mathrm{BC}$ cells MCF-7 to stimulate heparinase expression ${ }^{90}$. The results revealed that $\mathrm{BC}$ derived heparan sulfate (HS) could be carried by exosome particles, leading to upregulation of heparanase (HPSE) and HPSE2 expression of lymphocytes, which was highly relevant for cellular migration and tumor metastasis. Blomme et al. also showed that the cancer-overexpressed protein myoferlin was present in exosomes derived from $\mathrm{BC}$ cells to promote cell migration and invasion ${ }^{91}$. The depletion of myoferlin in cancer exosomes significantly modulated exosomal protein load, and reduced the capability to induce HUVEC migration and proliferation. Thrombospondin-1 (TSP1) is a multifunctional ECM glycoprotein, generally regulates the signaling pathways of $\mathrm{CD} 47, \mathrm{CD} 36$, and TGF- $\beta$ to promote tumor progression in various malignancies ${ }^{92}$. Cen et al. showed that BCderived exosomal TSP1 facilitated the trans-endothelial migration of $\mathrm{BC}$ cells by disrupting the intercellular integrity of endothelial cells, and by reducing the expression of intercellular junction proteins VE-cadherin and $\mathrm{ZO}-1$ both in vitro and in vivo ${ }^{93}$. 


\section{Exosomal proteins mediate colonization of targeted organ}

Nephronectin (NPNT) was the primary ligand for $\alpha 8 \beta 1$ integrin and was crucial in kidney development and had intricating roles in $\mathrm{BC}$ progression and metastasis. In 2018, Steigedal et al. showed that NPNT might be localized in mouse $\mathrm{BC}$-cell-derived exosomes and display the ability to increase adhesion and anchorage-independent growth in vitro in an integrin-dependent manner ${ }^{94}$. The disruption of the integrin-binding site within NPNT could regulate the capability of $\mathrm{mBC}$ cells to adhere and colonize in the lung. This study presumed that there was a link between NPNT-containing exosomes and enhanced metastatic capacity in vivo. Zhang et al. demonstrated that POPX2 was found to be associated with BC-cell invasiveness and lung colonization in the early stages of metastasis $^{95}$. Knockdown of POPX2 promoted exosome secretion to modulate the cytokine secretome including angiogenic proteins, such as fibroblast growth factor (FGF) and platelet-derived growth factor (PDGF), thus contributing to tumor angiogenesis at later stages of metastasis.

BCBM often induces neurological impairments by affecting cognitive and sensory functions and confer a poor prognosis, high mortality, and lack of effective therapy. A comprehensive understanding of tumorintrinsic properties and drivers of the crosstalk that allows $\mathrm{BC}$ cells to infiltrate the brain is critical to prevent and treat BCBM. BC-derived exosomes were essential in remodeling the brain microenvironment during metastatic colonization. The cell migration-inducing protein (CEMIP), also called KIAA1199, had increased expression in cancers and functioned as a regulator related to cell survival, growth, and invasion ${ }^{96}$. CEMIP was screened in exosomes released by viable brain metastatic tissues from breast and lung cancer patients and its high expression in human primary and metastatic tumors was obviously related to the accelerated metastatic progression and poor survival rate ${ }^{97}$. CEMIP was abundant in exosomes from additional orthotopic brain metastatic models of BC. It was interesting that tumor-derived exosomal CEMIP not only remodeled brain vasculature but also induced inflammatory brain vascular niches to support brain metastasis. Furthermore, the exosomal CEMIP pretreatment promoted brain metastatic colonization, regaining the ability of CEMIP-depleted cells to associate with brain vasculature.

Significant changes in protein expression have been observed during the differentiation and maturation of osteoclasts, which can be used as an emerging circulating biomarker for bone metastasis. The pathogenesis of bone metastasis depends on cross-communication between tumor cells and various stromal cells such as osteoblasts, osteoclasts, and mineralized bone matrix. The cytosolic protein L-plastin could be released in BC-derived exosomes $^{98}$. The $\mathrm{BC}$ exosomal L-plastin by $\mathrm{BC}$ cells mediated osteoclast activation and facilitated metastatic bone osteolysis, inferring the critical role of L-plastin in stimulating osteoclastogenesis and promoting osteolysis during $\mathrm{BC}$ bone metastasis. Integrin $\beta 3$ (ITGB3) belongs to a family of transmembrane proteins that integrate the ECM processes and participates in reprogramming tumor metabolism. ITGB3 knockdown led to a decreased cellular energy metabolism, suppressed tumor growth, impaired cytokinesis, and migration possibilities, and decreased vesicle trafficking ${ }^{99}$. It was speculated that the level of ITGB3 was upregulated in exosomes derived from the MDA-MB-231 in the skeletal metastasis model, and functioned as a crucial molecule in the formation of premetastatic niche adhesion by mediating local and distal effects via direct contact or exosomes.

\section{Exosomal metabolites}

Reprogrammed energy metabolism is a hallmark for tumor features. Energetic requirements and excretions exhibit huge unbalance in tumor cells to mediate tumor growth and metastasis through metabolite transfer and metabolic coupling. Emerging studies have demonstrated that the proteome and transcriptome of tumor metastasis are dynamically regulated by the metabolome. Metabolome-induced signaling cascades may drive tumor aggression and metastasis via diverse signal-regulating roles of metabolites in the complex process of metas$\operatorname{tasis}^{100}$. It proposed that ketone body production and reutilization could drive more enhanced $\mathrm{BC}$ growth and metastasis, as Martinez-Outschoorn et al. reported that ketogenic fibroblasts could modify MDA-MB-231 cells that overexpressed the enzyme required for ketone reutilization to be a more invasive phenotype ${ }^{101}$. Similarly, Ko et al. showed that energy-rich glutamine was an essential molecular from autophagic fibroblasts to favor $\mathrm{BC}$-cell mitochondrial activity, and encouraged a pernicious cycle of catabolism in the tumor stroma and anabolic tumor cell dissemination ${ }^{102}$. Exosomes are efficient carriers to encapsulate metabolites, including lipids, lactate, glutamate, acetate, stearate, palmitate, and amino acids $^{103}$. For example, Puhka et al. implemented metabolomic analysis in the urinary exosome-containing extracellular vesicles from the prostate patients, indicating that the levels of glucuronate, D-ribose 5-phosphate, and isobutyryl-L-carnitine were significantly lower in prostatectomy samples than healthy control ${ }^{104}$. This study was potential evidence that the exosomal cargoes were far more complicated and contained various types of metabolites. Exosome-derived metabolites might respond to the extracellular environment and might be transporters to fuel rapid cell growth and proliferation.

Lipids are involved in exosome biosynthesis and are key components of exosomal membranes. Specifically, 
exosomes are rich in lipids such as sphingomyelin, ceramide, phosphatidylserine, cholesterol, and saturated fatty acids, and most of them are located on membrane ${ }^{105}$. Compared with their parental cells, specific lipids are located in exosomes and therefore have the potential to serve as identification markers ${ }^{106}$. Docosahexaenoic acid (DHA) is a long-chain, highly unsaturated omega 3 fatty acid with anti-angiogenesis effects. In vitro assay, exogenous DHA treatment downregulated the expression level of pro-angiogenic genes and miRNA (upregulated miR-101, miR-199, miR-342, and downregulated miR-382, miR-21) in MDA-MB-231 cell and their exosomes ${ }^{107}$. Some studies have verified that different mechanisms of exosomal lipids and the variability are involved in cancer diseases. Fatty acid encapsulated in tumor-derived exosomes could promote immune evasion by inducing dysfunctional dendritic cells $(\mathrm{DC})^{108}$. The cholesterol metabolite 27Hydroxycholesterol (27-OHC) was involved in proliferation and metastasis in estrogen receptor-positive $(\mathrm{ER}+) \mathrm{BC}$ via the ER receptor and liver $\mathrm{X}$ receptor (LXR), respectively ${ }^{109}$. Compared with exosomes derived from estrogen receptor-negative (ER-) BC-cell line (MDA-MB-231) and other control, the exosomal 27-OHC from $\mathrm{ER}+\mathrm{BC}$-cell line (MCF-7) were significantly increased ${ }^{110}$. Thus, lowering circulating cholesterol levels like 27-OHC may be a useful strategy to prevent and/or treat $\mathrm{BC}$. However, the value of exosomal 27-OHC in $\mathrm{BC}$ diagnosis was still lacking direct confirmation.

To sum up, these molecular metabolites are also encapsulated and shuttled to adjacent cells via $\mathrm{BC}$-derived exosomes. At present, most researches focus on exosomes containing certain proteins and RNAs. However, although many metabolites participate in $\mathrm{BC}$ progression, it is a pity that there are few studies about the metabolites carried by tumor-derived exosomes in BC. Generally speaking, the studies on the role of exosomal nucleic acids and proteins relatively are much more than metabolites in the tumor progression, partly because nucleic acids and proteins possess more genetic specificity to regulate recipient cell behaviors via exosome-mediated transportation. More studies are needed to decipher the role of exosomal lipids and amino acid metabolism to regulate tumor progression.

\section{BC-derived exosomes in BC metastasis immune regulation}

$\mathrm{BC}$ cells generate an immunosuppressed TME, which is a very highly intricate and heterogeneous system, incorporating cancer cells, CAFs, endothelial cells, immune cells, ECM, and various cytokines. TME is associated with induction of proliferation, angiogenesis, apoptosis inhibition and immune system suppression, and drug resistance ${ }^{111}$. Especially, induction of antitumor immunity to resist immune suppression is one of the most breathtaking strategies for therapeutic use for tumor therapy. Escaping immune surveillance is a hallmark and prerequisite for the establishment of a permissive environment in the secondary organs of the primary tumor, thereby enabling metastasis and sustaining tumor progression. In the immune system, exosomes are fundamental mediators secreted by each immune cell type to fulfill its function and promote inflammation or tolerance. The activities of exosomes affect multiple immunological behaviors, including modulating antigen presentation, immune activation and suppression, immune surveillance, and intercellular communication ${ }^{112}$. It is well known that tumor-derived exosomes can exert detrimental effects on the immune system by impairing specific $\mathrm{T}$-cell immunity and bias innate immune cells toward a tumor-promoting phenotype. Clearly, tumor-derived exosomes impact recipient immune cells with the complex nature of those interactions and the molecular drivers to propel tumor metastasis. The inhibition of tumor-derived exosome uptake or carried components is of value to restore function in immune-suppressed cells.

$\mathrm{BC}$ exosomes travel to specific homing niches dynamically modify gene expression and molecular architectures to mediate immune lymphocyte migration, host defense, and tumor metastasis ${ }^{113}$. Hoshino et al. verified that $\mathrm{BC}$ exosomes expressing high levels of $\alpha \nu \beta 5$ were distributed to liver tissue containing FN-enriched ECM, whereas those with high levels of integrin $\alpha 6 \beta 4$ were distributed to the lung tissue containing ECM enriched with laminin ${ }^{114}$. The exogenously fluorescently labeled exosomes of highly-metastatic murine $\mathrm{BC}$ cells were recruited predominantly to mice lung where $\mathrm{BC}$ metastases were frequent, speculating $\mathrm{BC}$ exosome accumulation made the lung environment more prone to metastatic tumor colonization ${ }^{115}$. Moreover, the BC-derived exosome showed a stimulative effect on myeloid-derived suppressor cells (MDSC) accumulation and a direct suppressive effect on T-cell proliferation and NK cell cytotoxicity, and hence likely weakened the antitumor immune response in premetastatic niches. Therefore, the tissue-specificity of BCderived exosome accumulation contributed to immune suppression and increased metastatic colonization. Jiang et al. proposed that BC exosomal miR-9 and miR-181a were upregulated in early-stage MDSCs to promote the early-stage MDSCs amplification and T-cell immunity suppression, leading to promote tumor growth ${ }^{116}$. This process was mediated via JAK/STAT signaling pathway activation by targeting SOCS3 and PIAS3, respectively. Deng et al. found that tumor cell interaction with tumorassociated leukocytes was required for induction of tumor-exosomal $\mathrm{FN}^{77}$. The tumor-derived exosomal FN promoted 4T1 breast tumor invasion and metastasis in vivo, which could be regulated by CD25+ cells and Gr$1+$ cells by recruiting FN into exosomes. 
Since tumor-derived exosomes could target immune cells to induce an immunosuppressive tissue environment, providing the potential of serving as targets for novel anticancer therapies for $\mathrm{BC}$. The engineered $\mathrm{BC}$ exosome modified with overexpressed miRNAs had potential anticancer effects as cell-free vaccine for BC treatment ${ }^{117}$. The $\mathrm{BC}$ exosome manipulated Let-7i, miR142, and miR-155 synergistically and efficiently induced the DC maturation and promote tumor escape ${ }^{117}$. BC exosomes carried programmed death-ligand 1 (PD-L1) were highly immunosuppressive in BC TME. TGF- $\beta$ acted as a promoter of exosomal PD-L1 to promote CD8+ Tcell dysfunction. This result proclaimed that TGF- $\beta$ espoused tumor-derived exosomal PD-L1 to attenuate phosphorylation of Src family proteins of activated CD8+ $\mathrm{T}$ cells and promote CD8+ T-cell dysfunction ${ }^{118}$. These results all confirmed the significance for the roles of tumor-derived exosomes in communicating with immune cells, involved in DC differentiation and maturation, CD8 $+\mathrm{T}$-cell dysfunction, and regulatory immune cells like MDSCs and Tregs. More comprehensive proteomic and RNA profiling studies of BC-derived exosomes could help identify key exosome contents that drive immune regulation.

\section{Conclusions and expectations}

Exosomes have gained extensive attention in cancer due to the multifaceted roles such as remodeling TME, reshaping tumor progression, and conferring therapy resistance. The current studies have partially elucidated the behaviors and mechanisms of $\mathrm{BC}$-derived exosomes in reprogramming $\mathrm{BC}$ metastasis, which principally ascribed to the content of tumor exosome and the bioactive cargoes encapsulated in exosomes. In this review, we have found that the exosomal miRNA (miR-146a, miR-19103p, miR-9, and miR-155, etc.), IncRNA (lncRNAGS1600G8.5, hsa-circRNA-0088088, etc.), circRNA, protein (MTA1, UCHL1, AnxA2, TSP1, etc.), lipid (27-OHC), were the main active components being studied in $\mathrm{BC}$ metastasis, as listed in Table 1 . The organotypic metastasis induced by these $\mathrm{BC}$ exosomal components is achieved by regulating pre-metastatic niche formation, EMT, vascular permeability, angiogenesis, and colonization (Fig. 3).

However, there still are some challenges deserving of our attention in this area. Firstly, the BC metastasis ability is closely related to the molecular classification of $\mathrm{BC}$. For example, TNBC and basal-like BC are of highly tumor metastasis characteristics and the prognosis is better for $\mathrm{ER}+\mathrm{BC}$. In existing studies, the important relationship between exosomal cargoes and metastasis of specific $\mathrm{BC}$ subtype were not clinically emphasized and deserved further investigation. Secondly, TME is a complicated entity composed of multiple cell types and cell-secreted factors. That means, exosomes originated from different cell types orchestrate the tumor cell metastasis and the metastasis is the ending caused by these exosomal components and others synergistically. Therefore, it is difficult to judge the exosomes from which cell types occupy the leading position for $\mathrm{BC}$ metastasis. Thirdly, exosomal ncRNAs are evolving as important mediators in the interplay between the $\mathrm{BC}$ cells and TME. Although there are relatively many studies on miRNA, the studies on IncRNA and circRNA are still relatively insufficient. Besides, some ncRNA studies only explored the expression level by sequencing and PCR verification on tissue samples or cell lines, but lacking further investigation on metastasis mechanism. Fourthly, tumorderived exosomes carry a wide range of nucleic acid and proteins, lipids, that can reflect the molecular contents of the parental cells. However, the abundance of these substances in exosomes is different from the expression level of parent cells, indicating that these substances are selectively encapsulated in exosomes in different cell types, including $\mathrm{BC}$ cells. And this phenomenon of selective encapsulation mechanism needs to be further addressed. Lastly, successful colonization of $\mathrm{mBC}$ cells requires reciprocal communications with stroma cells, endothelial cells, and local immune cells. The role and possible alteration of immune regulation in establishing pre-metastatic niches in future metastatic organs should be investigated.

For future perspectives, it will be meaningful to take consideration of tumor-derived exosomal biomarkers for $\mathrm{BC}$ diagnosis and therapy. Firstly, exosomes are promising candidates as biomarkers for early detection of $\mathrm{BC}$ and their prognosis. The conventional screening methods for $\mathrm{BC}$ including mammography, ultrasonography, PET-CT, and MRI, are usually costly and time consuming. The liquid biopsy is a classical and quick method by detecting various body fluids such as plasma, serum, urea, saliva, and tears. To some extent, exosomes loaded with specific bioactive molecules with differential expression in the above body fluids can represent the BC course and metastasis, thus posing the potential with sensitivity and specificity to detect early metastasis and predict prognosis for BC patients by liquid biopsy. Secondly, undoubtedly, tumor-derived exosomes are potential therapeutic targets in BC therapy, including metastasis intervention, drug resistance, and drug delivery. Blocking the release or the key components of exosomes associated with metastasis represents a new approach for developing anti-BC therapeutic agents, which might be used alone or in conjunction with traditional therapies.

Further works are needed to illuminate the potential mechanisms of exosomal components in $\mathrm{BC}$ metastasis. The profound understanding of tumor-exosomal components in reshaping the properties of $\mathrm{BC}$ metastasis, will 


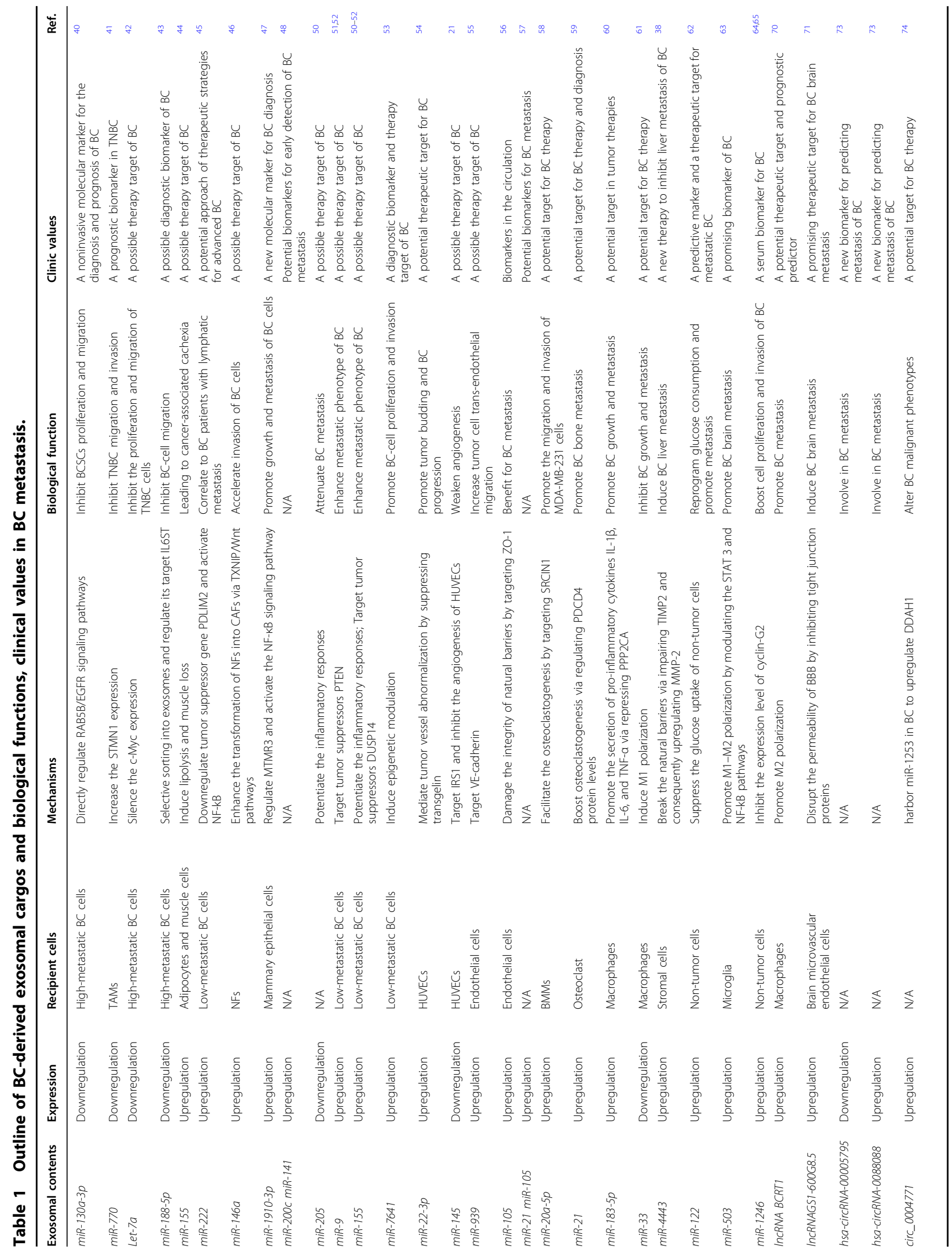




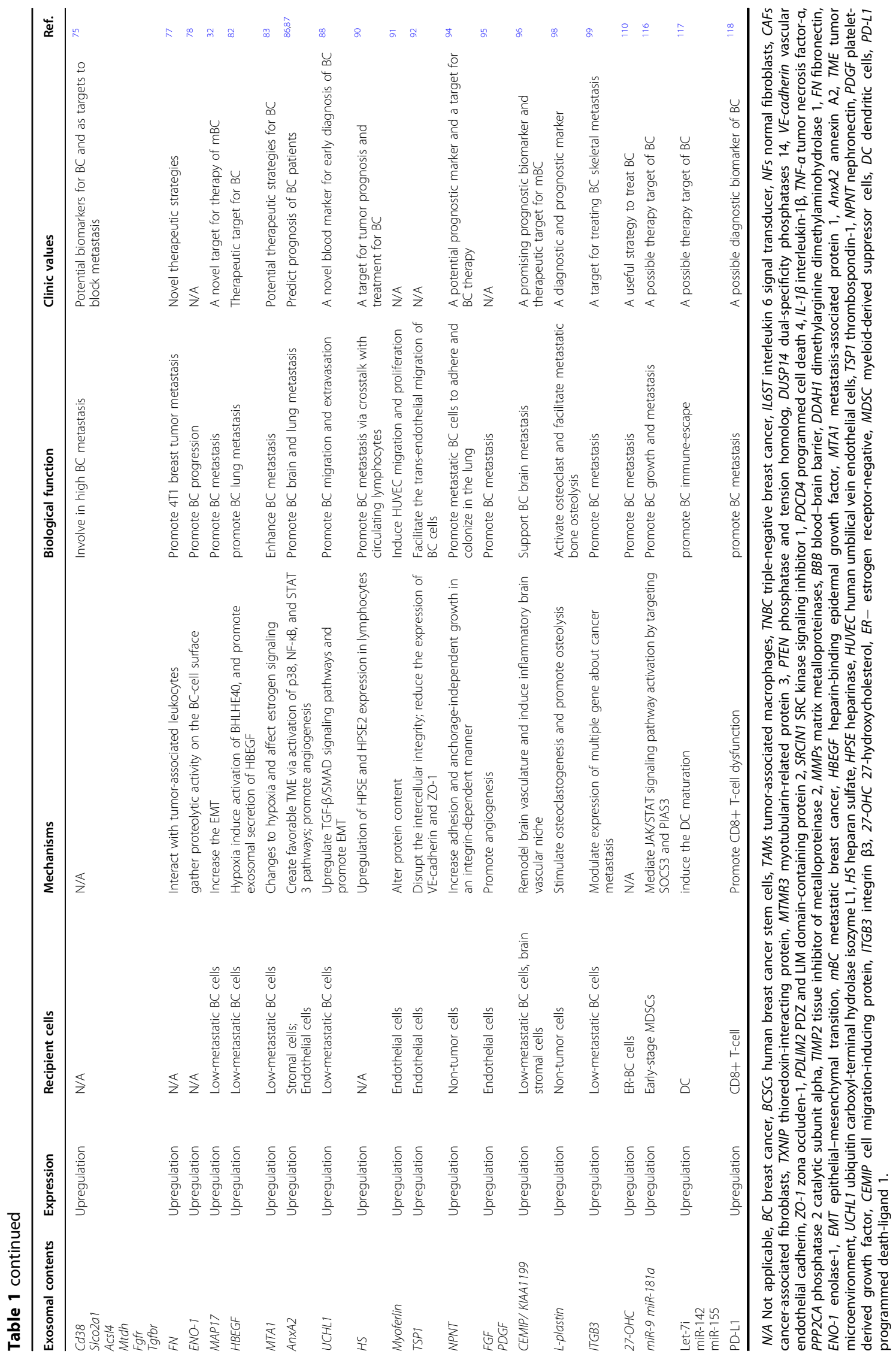




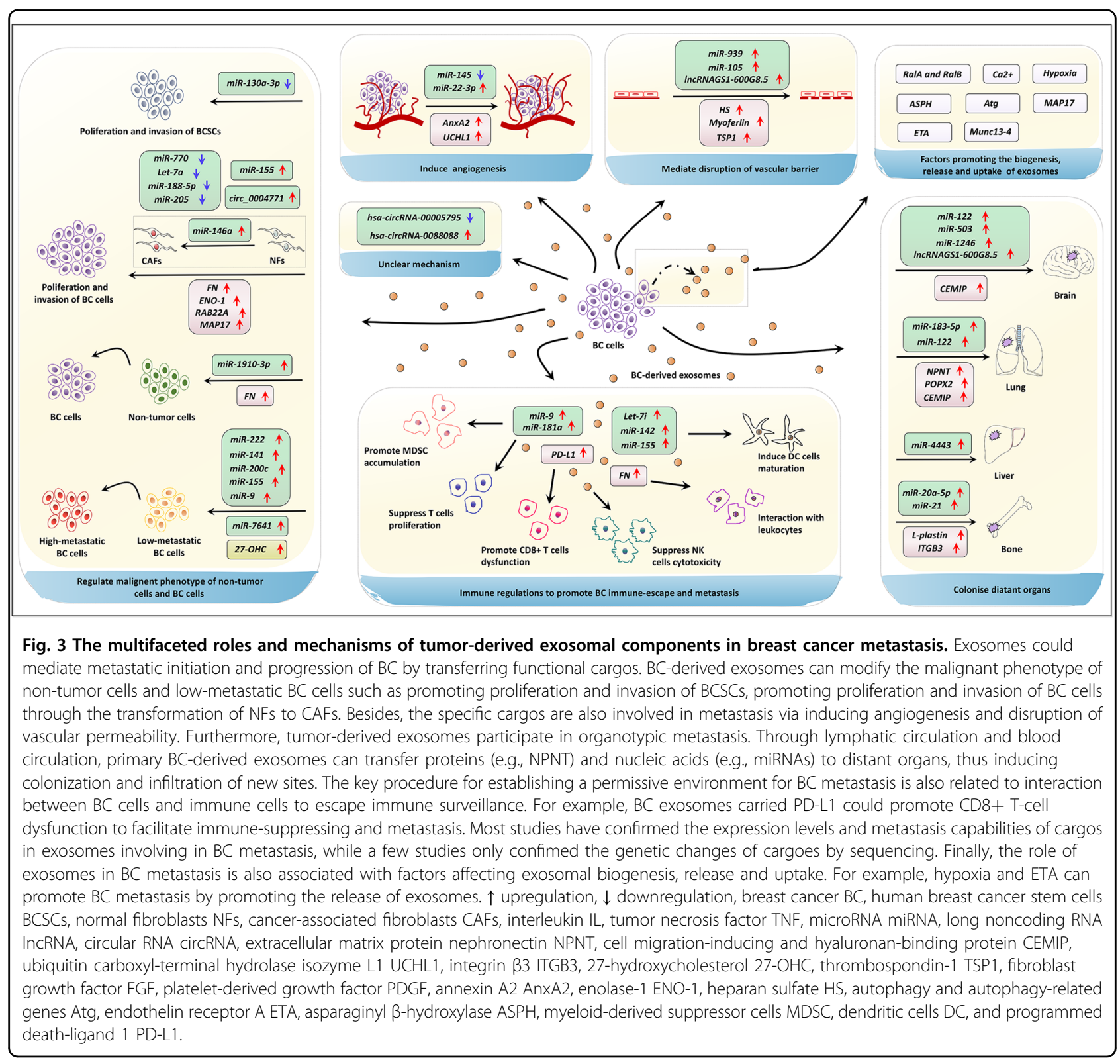

undoubtedly provide novel insights for developing diagnosis and therapy strategies.

\section{Acknowledgements}

Not applicable.

\section{Funding}

This work was supported by China Guanghua Science and Technology Foundation (grant number: 2019JZXM001) and Wuhan Science and Technology Bureau (grant number: 2020020601012241).

\section{Author contributions}

Y.T., X.L., and W.L. performed literature investigation and wrote the manuscript. H.W., Q.Z., and Y.W. conceived the project and designed the outline. W.H., C.Z., M.X., Y.Y., D.W., and Y.W. edited and revised the paper. All authors have reviewed the paper and all approved of the final version.
Data availability

Not applicable.

Competing interests

The authors declare no competing interests.

\section{Consent for publication}

All authors have provided their consent for publication.

Ethical approval and consent to participate

Not applicable.

\section{Publisher's note}

Springer Nature remains neutral with regard to jurisdictional claims in published maps and institutional affiliations. 
Received: 4 March 2021 Revised: 11 May 2021 Accepted: 11 May 2021 Published online: 26 May 2021

\section{References}

1. Siegel, R. L., Miller, K. D. \& Jemal, A. Cancer statistics, 2020. Ca. Cancer J. Clin. 70, 7-30 (2020).

2. Koboldt, D. C. et al. Comprehensive molecular portraits of human breast tumours. Nature 490, 61-70 (2012).

3. Liang, Y., Zhang, H., Song, X. \& Yang, Q. Metastatic heterogeneity of breast cancer: Molecular mechanism and potential therapeutic targets. Semin. Cancer Biol. 60, 14-27 (2020).

4. Esteva, F. J., Hubbard-Lucey, V. M., Tang, J. \& Pusztai, L. Immunotherapy and targeted therapy combinations in metastatic breast cancer. Lancet Oncol. 20, e175-e186 (2019).

5. Pegtel, D. M. \& Gould, S. J. Exosomes. Annu. Rev. Biochem. 88, 487-514 (2019).

6. H. Rashed, M. et al. Exosomes: from garbage bins to promising therapeutic targets. Int. J. Mol. Sci. 18, 538 (2017).

7. Cheng, N. et al. Recent advances in biosensors for detecting cancer-derived exosomes. Trends Biotechnol. 37, 1236-1254 (2019).

8. Chen, F. et al. The functional roles of exosomes-derived long non-coding RNA in human cancer. Cancer Biol. Ther. 20, 583-592 (2019).

9. Kok, V. C. \& Yu, C. C. Cancer-derived exosomes: their role in cancer biology and biomarker development. Int. J. Nanomed. 15, 8019-8036 (2020).

10. Chen, W., Hoffmann, A. D., Liu, H. \& Liu, X. Organotropism: new insights into molecular mechanisms of breast cancer metastasis. npj Precis. Oncol. 2, 4 (2018).

11. Giordano, C. et al. The biology of exosomes in breast cancer progression: dissemination, immune evasion and metastatic colonization. Cancers $\mathbf{1 2}$ 2179 (2020).

12. Rodríguez, M. et al. Exosomes enriched in stemness/metastatic-related mRNAS promote oncogenic potential in breast cancer. Oncotarget $\mathbf{6}$, 40575-40587 (2015).

13. Piao, Y. J. et al. Breast cancer cell-derived exosomes and macrophage polarization are associated with lymph node metastasis. Oncotarget $\mathbf{9}$, 7398-7410 (2018).

14. Wortzel, I., Dror, S., Kenific, C. M. \& Lyden, D. Exosome-mediated metastasis: communication from a distance. Dev. Cell 49, 347-360 (2019).

15. Patel, J. S. et al. Non-coding RNA as mediators in microenvironment-breast cancer cell communication. Cancer Lett. 380, 289-295 (2016).

16. Steinbichler, T. B., Dudás, J., Riechelmann, H. \& Skvortsova, I.-I. The role of exosomes in cancer metastasis. Semin. Cancer Biol. 44, 170-181 (2017).

17. Wong, G. L., Abu Jalboush, S. \& Lo, H.-W. Exosomal MicroRNAs and organotropism in breast cancer metastasis. Cancers 12, 1827 (2020).

18. Daßler-Plenker, J., Küttner, V. \& Egeblad, M. Communication in tiny packages: exosomes as means of tumor-stroma communication. Biochim. Biophys. Acta Rev. Cancer 1873, 188340 (2020).

19. Wang, H., Lu, Z. \& Zhao, X. Tumorigenesis, diagnosis, and therapeutic potential of exosomes in liver cancer. J. Hematol. Oncol. 12, 133 (2019).

20. Ghoroghi, S. et al. Ral GTPases promote breast cancer metastasis by controlling biogenesis and organ targeting of exosomes. Elife 10, 1-29 (2021).

21. Pan, S. et al. STIM1 promotes angiogenesis by reducing exosomal miR-145 in breast cancer MDA-MB-231 cells. Cell Death Dis. 12, 38 (2021).

22. Terry, S. et al. Role of hypoxic stress in regulating tumor immunogenicity, resistance and plasticity. Int. J. Mol. Sci. 19, 3044 (2018).

23. Shao, $\mathrm{C}$. et al. Role of hypoxia-induced exosomes in tumor biology. Mol. Cancer 17, 120 (2018).

24. King, H. W., Michael, M. Z. \& Gleadle, J. M. Hypoxic enhancement of exosome release by breast cancer cells. BMC Cancer 12, 421 (2012).

25. Wang, T. et al. Hypoxia-inducible factors and RAB22A mediate formation of microvesicles that stimulate breast cancer invasion and metastasis. Proc. Natl Acad. Sci. 111, E3234-E3242 (2014).

26. Shimoda, M. et al. Endocrine sensitivity of estrogen receptor-positive breast cancer is negatively correlated with aspartate- $\beta$-hydroxylase expression. Cancer Sci. 108, 2454-2461 (2017).

27. Lin, Q. et al. ASPH-notch axis guided exosomal delivery of prometastatic secretome renders breast cancer multi-organ metastasis. Mol. Cancer 18, 156 (2019).
28. Wang, L. et al. Expression of autophagy-related proteins ATG5 and FIP200 predicts favorable disease-free survival in patients with breast cancer. Biochem. Biophys. Res. Commun. 458, 816-822 (2015).

29. Guo, H. et al. Atg5 disassociates the V1V0-ATPase to promote exosome production and tumor metastasis independent of canonical macroautophagy. Dev. Cell 43, 716-730.e7 (2017).

30. Im, E.-J. et al. Sulfisoxazole inhibits the secretion of small extracellular vesicles by targeting the endothelin receptor A. Nat. Commun. 10, 1387 (2019).

31. Messenger, S. W., Woo, S. S., Sun, Z. \& Martin, T. F. J. A Ca2+-stimulated exosome release pathway in cancer cells is regulated by Munc13-4. J. Cell Biol. 217, 2877-2890 (2018)

32. García-Heredia, J. M. et al. Breast tumor cells promotes the horizontal propagation of EMT, stemness, and metastasis by transferring the MAP17 protein between subsets of neoplastic cells. Oncogenesis 9, 96 (2020).

33. Esposito, C. L. et al. Identification of a novel RNA aptamer that selectively targets breast cancer exosomes. Mol. Ther. Nucleic Acids 23, $982-994$ (2021).

34. Gourlay, J. et al. The emergent role of exosomes in glioma. J. Clin. Neurosci. 35, 13-23 (2017)

35. Gurunathan, S., Kang, M. H. \& Kim, J. H. A comprehensive review on factors influences biogenesis, functions, therapeutic and clinical implications of exosomes. Int. J. Nanomed. 16, 1281-1312 (2021).

36. Syn, N., Wang, L., Sethi, G., Thiery, J.P. \& Goh, B.-C. Exosome-mediated metastasis: from epithelial-mesenchymal transition to escape from immunosurveillance. Trends Pharmacol. Sci. 37, 606-617 (2016).

37. Vahidian, F. et al. MicroRNAs and breast cancer stem cells: potential role in breast cancer therapy. J. Cell. Physiol. 234, 3294-3306 (2019).

38. Wang, J. et al. Microenvironment-induced TIMP2 loss by cancer-secreted exosomal miR-4443 promotes liver metastasis of breast cancer. J. Cell. Physiol. 235, 5722-5735 (2020).

39. Melo, S. A. et al. Cancer exosomes perform cell-independent MicroRNA biogenesis and promote tumorigenesis. Cancer Cell 26, 707-721 (2014).

40. Kong, X., Zhang, J., Li, J., Shao, J. \& Fang, L. MiR-130a-3p inhibits migration and invasion by regulating RAB5B in human breast cancer stem cell-like cells. Biochem. Biophys. Res. Commun. 501, 486-493 (2018).

41. Li, Y. et al. MiR-770 suppresses the chemo-resistance and metastasis of triple negative breast cancer via direct targeting of STMN1. Cell Death Dis. 9, 14 (2018).

42. Du, J., Fan, J. J., Dong, C., Li, H. T. \& Ma, B. L. Inhibition effect of exosomesmediated Let-7a on the development and metastasis of triple negative breast cancer by down-regulating the expression of c-Myc. Eur. Rev. Med. Pharmacol. Sci. 23, 5301-5314 (2019).

43. Wang, M. et al. miR-188-5p suppresses cellular proliferation and migration via IL6ST: a potential noninvasive diagnostic biomarker for breast cancer. J. Cell. Physiol. 235, 4890-4901 (2020).

44. Wu, Q. et al. Breast cancer-released exosomes trigger cancer-associated cachexia to promote tumor progression. Adipocyte 8, 31-45 (2019).

45. Ding, J. et al. Exosome-mediated miR-222 transferring: an insight into NF-KBmediated breast cancer metastasis. Exp. Cell Res. 369, 129-138 (2018).

46. Yang, S. S. et al. Breast cancer-derived exosomes regulate cell invasion and metastasis in breast cancer via miR-146a to activate cancer associated fibroblasts in tumor microenvironment. Exp. Cell Res. 391, 111983 (2020).

47. Wang, B. et al. Exosomal miR-1910-3p promotes proliferation, metastasis, and autophagy of breast cancer cells by targeting MTMR3 and activating the NFKB signaling pathway. Cancer Lett. 489, 87-99 (2020).

48. Zhang, $\mathrm{G}$. et al. MicroRNA-200C and microRNA- 141 are regulated by a FOXP3-KAT2B axis and associated with tumor metastasis in breast cancer. Breast Cancer Res. 19, 73 (2017).

49. Santos, J. C. et al. Exosome-mediated breast cancer chemoresistance via miR155 transfer. Sci. Rep. 8, 829 (2018).

50. Gorczynski, R. M., Zhu, F., Chen, Z., Kos, O. \& Khatri, I. A comparison of serum miRNAs influencing metastatic growth of EMT6 vs 4THM tumor cells in wildtype and CD200R1KO mice. Breast Cancer Res. Treat. 162, 255-266 (2017).

51. Kia, V., Paryan, M., Mortazavi, Y., Biglari, A. \& Mohammadi-Yeganeh, S. Evaluation of exosomal miR-9 and miR-155 targeting PTEN and DUSP14 in highly metastatic breast cancer and their effect on low metastatic cells. J. Cell. Biochem. 120, 5666-5676 (2019).

52. kia, V., Mortazavi, Y., Paryan, M. \& Biglari, A. Exosomal miRNAs from highly metastatic cells can induce metastasis in non-metastatic cells. Life Sci. 220, 162-168 (2019).

53. Shen, S. et al. Cancer-derived exosomal miR-7641 promotes breast cancer progression and metastasis. Cell Commun. Signal. 19, 20 (2021). 
54. Feng, $Y$. et al. Tumor cell-secreted exosomal miR-22-3p inhibits transgelin and induces vascular abnormalization to promote tumor budding. Mol. Ther. 29, 1-16 (2021).

55. Di Modica, M. et al. Breast cancer-secreted miR-939 downregulates VEcadherin and destroys the barrier function of endothelial monolayers. Cancer Lett. 384, 94-100 (2017).

56. Zhou, W. et al. Cancer-secreted miR-105 destroys vascular endothelial barriers to promote metastasis. Cancer Cell 25, 501-515 (2014).

57. Rodríguez-Martínez, A. et al. Exosomal miRNA profile as complementary tool in the diagnostic and prediction of treatment response in localized breast cancer under neoadjuvant chemotherapy. Breast Cancer Res. 21, 21 (2019).

58. Guo, L. et al. Breast cancer cell-derived exosomal miR-20a-5p promotes the proliferation and differentiation of osteoclasts by targeting SRCIN1. Cancer Med. 8, 5687-5701 (2019).

59. Yuan, X. et al. Breast cancer exosomes contribute to pre-metastatic niche formation and promote bone metastasis of tumor cells. Theranostics $\mathbf{1 1}$ 1429-1445 (2021).

60. Guo, J. et al. Mouse 4T1 breast cancer cell-derived exosomes induce proinflammatory cytokine production in macrophages via miR-183. J. Immunol. 205, 2916-2925 (2020).

61. Moradi-Chaleshtori, M., Bandehpour, M., Heidari, N., Mohammadi-Yeganeh, S. \& Mahmoud Hashemi, S. Exosome-mediated miR-33 transfer induces M1 polarization in mouse macrophages and exerts antitumor effect in 4T1 breast cancer cell line. Int. Immunopharmacol. 90, 107198 (2021).

62. Fong, M. Y. et al. Breast-cancer-secreted miR-122 reprograms glucose metabolism in premetastatic niche to promote metastasis. Nat. Cell Biol. 17 183-194 (2015).

63. Xing, F. et al. Loss of XIST in breast cancer activates MSN-c-Met and reprograms microglia via exosomal miRNA to promote brain metastasis. Cancer Res. 78, 4316-4330 (2018).

64. Sharma, S. et al. Ca2+ and CACNA1H mediate targeted suppression of breast cancer brain metastasis by AM RF EMF. EBioMedicine 44, 194-208 (2019).

65. Li, X. J., Ren, Z. J., Tang, J. H. \& Yu, Q. Exosomal MicroRNA MiR-1246 promotes cell proliferation, invasion and drug resistance by targeting CCNG2 in breast cancer. Cell. Physiol. Biochem. 44, 1741-1748 (2018).

66. Xu, S., Kong, D., Chen, Q., Ping, Y. \& Pang, D. Oncogenic long noncoding RNA landscape in breast cancer. Mol. Cancer 16, 129 (2017).

67. Wang, S. et al. JAK2-binding long noncoding RNA promotes breast cancer brain metastasis. J. Clin. Investig. 127, 4498-4515 (2017).

68. $\mathrm{Ni}, \mathrm{C}$. et al. Breast cancer-derived exosomes transmit IncRNA SNHG16 to induce CD73+ $\gamma \delta 1$ Treg cells. Signal Transduct. Target. Ther. 5, 41 (2020).

69. Feng, T. et al. High throughput sequencing identifies breast cancer-secreted exosomal LncRNAs initiating pulmonary pre-metastatic niche formation. Gene 710, 258-264 (2019).

70. Liang, Y. et al. LncRNA BCRT1 promotes breast cancer progression by targeting miR-1303/PTBP3 axis. Mol. Cancer 19, 85 (2020).

71. Lu, Y., Chen, L., Li, L. \& Cao, Y. Exosomes derived from brain metastatic breast cancer cells destroy the blood-brain barrier by carrying IncRNA GS1-600G8.5. Biomed. Res. Int. 2020, 7461727 (2020).

72. Wang, J. et al. Circular RNA expression in exosomes derived from breast cancer cells and patients. Epigenomics 11, 411-421 (2019).

73. Yang, S. et al. Identification of circRNA-miRNA networks for exploring an underlying prognosis strategy for breast cancer. Epigenomics 12, 101-125 (2020)

74. Ding, $X$., Zheng, J. \& Cao, M. Circ_0004771 accelerates cell carcinogenic phenotypes via suppressing miR-1253-mediated DDAH1 inhibition in breast cancer. Cancer Manag. Res. 13, 1-11 (2021).

75. Gangoda, L. et al. Proteomic profiling of exosomes secreted by breast cancer cells with varying metastatic potential. Proteomics 17, 1600370 (2017).

76. Itagaki, K. et al. Exposure of the cryptic de-adhesive site FNIII14 in fibronectin molecule and its binding to membrane-type eEF1A induce migration and invasion of cancer cells via $\beta 1$-integrin inactivation. Am. J. Cancer Res. 10 3990-4004 (2020).

77. Deng, Z et al. Tumor cell cross talk with tumor-associated leukocytes leads to induction of tumor exosomal fibronectin and promotes tumor progression. Am. J. Pathol. 180, 390-398 (2012)

78. Didiasova, M. et al. STIM1/ORAl1-mediated Ca 2+ influx regulates enolase-1 exteriorization. J. Biol. Chem. 290, 11983-11999 (2015).
79. Sun, L. et al. Regulation of RAB22A by miR-193b inhibits breast cancer growth and metastasis mediated by exosomes. Int. J. Oncol. 53, 2705-2714 (2018).

80. Lu, X. \& Kang, Y. Hypoxia and hypoxia-inducible factors: master regulators of metastasis. Clin. Cancer Res. 16, 5928-5935 (2010).

81. Meng, W., Hao, Y., He, C., Li, L. \& Zhu, G. Exosome-orchestrated hypoxic tumor microenvironment. Mol. Cancer 18, 57 (2019).

82. Sethuraman, A. et al. BHLHE40 confers a pro-survival and pro-metastatic phenotype to breast cancer cells by modulating HBEGF secretion. Breast Cancer Res. 20, 117 (2018).

83. Hannafon, B. N. et al. Metastasis-associated protein 1 (MTA1) is transferred by exosomes and contributes to the regulation of hypoxia and estrogen signaling in breast cancer cells. Cell Commun. Signal. 17, 13 (2019).

84. Ekström, E. J. et al. WNT5A induces release of exosomes containing proangiogenic and immunosuppressive factors from malignant melanoma cells. Mol. Cancer 13, 88 (2014).

85. Liu, W. \& Hajjar, K. A. The annexin A2 system and angiogenesis. Biol. Chem. 397, 1005-1016 (2016)

86. Maji, S. et al. Exosomal annexin II promotes angiogenesis and breast cancer metastasis. Mol. Cancer Res. 15, 93-105 (2017).

87. Chaudhary, P. et al. Serum exosomal-annexin A2 is associated with AfricanAmerican triple-negative breast cancer and promotes angiogenesis. Breast Cancer Res. 22, 31 (2020).

88. Liu, S. et al. Deubiquitinase activity profiling identifies UCHL1 as a candidate oncoprotein that promotes TGF $\beta$-induced breast cancer metastasis. Clin Cancer Res. 26, 1460-1473 (2020).

89. Azzi, S., Hebda, J. K. \& Gavard, J. Vascular permeability and drug delivery in cancers. Front. Oncol. 3, 211 (2013).

90. Theodoro, T. R. et al. Crosstalk between tumor cells and lymphocytes modulates heparanase expression. J. Transl. Med. 17, 103 (2019).

91. Blomme, A. et al. Myoferlin is a novel exosomal protein and functional regulator of cancer-derived exosomes. Oncotarget 7, 83669-83683 (2016)

92. Kamijo, $\mathrm{H}$. et al. Thrombospondin-1 promotes tumor progression in cutaneous T-cell lymphoma via CD47. Leukemia 34, 845-856 (2020).

93. Cen, J. et al. Exosomal thrombospondin-1 disrupts the integrity of endothelial intercellular junctions to facilitate breast cancer cell metastasis. Cancers 11, 1946 (2019).

94. Steigedal, T. S. et al. Nephronectin is correlated with poor prognosis in breast cancer and promotes metastasis via its integrin-binding motifs. Neoplasia $\mathbf{2 0}$, 387-400 (2018).

95. Zhang, S. et al. Phosphatase POPX2 exhibits dual regulatory functions in cancer metastasis. J. Proteome Res. 16, 698-711 (2017).

96. Deroyer, C. et al. CEMIP (KIAA1199) induces a fibrosis-like process in osteoarthritic chondrocytes. Cell Death Dis. 10, 103 (2019).

97. Rodrigues, G. et al. Tumour exosomal CEMIP protein promotes cancer cell colonization in brain metastasis. Nat. Cell Biol. 21, 1403-1412 (2019).

98. Tiedemann, K. et al. Exosomal release of L-plastin by breast cancer cells facilitates metastatic bone osteolysis. Transl. Oncol. 12, 462-474 (2019).

99. Kovacheva, M., Zepp, M., Berger, S. \& Berger, M. R. Conditional knockdown of integrin beta-3 reveals its involvement in osteolytic and soft tissue lesions of breast cancer skeletal metastasis. J. Cancer Res. Clin. Oncol. 147, 361-371 (2021).

100. Liu, J. Y. \& Wellen, K. E. Advances into understanding metabolites as signaling molecules in cancer progression. Curr. Opin. Cell Biol. 63, 144-153 (2020).

101. Martinez-Outschoorn, U. E. et al. Ketone body utilization drives tumor growth and metastasis. Cell Cycle 11, 3964-3971 (2012).

102. Ko, Y.-H. et al. Glutamine fuels a vicious cycle of autophagy in the tumor stroma and oxidative mitochondrial metabolism in epithelial cancer cells. Cancer Biol. Ther. 12, 1085-1097 (2011).

103. Kalluri, R. \& LeBleu, V. S. The biology, function, and biomedical applications of exosomes. Science 367, eaau6977 (2020).

104. Puhka, M. et al. Metabolomic profiling of extracellular vesicles and alternative normalization methods reveal enriched metabolites and strategies to study prostate cancer-related changes. Theranostics 7, 3824-3841 (2017).

105. Record, M., Carayon, K. Poirot, M. \& Silvente-Poirot, S. Exosomes as new vesicular lipid transporters involved in cell-cell communication and various pathophysiologies. Biochim. Biophys. Acta Mol. Cell Biol. Lipids 1841, 108-120 (2014).

106. Skotland, T., Sandvig, K. \& Llorente, A. Lipids in exosomes: current knowledge and the way forward. Prog. Lipid Res. 66, 30-41 (2017). 
107. Aslan, C. et al. Docosahexaenoic acid (DHA) inhibits pro-angiogenic effects of breast cancer cells via down-regulating cellular and exosomal expression of angiogenic genes and microRNAs. Life Sci. 258, 118094 (2020).

108. Yin, $X$. et al. PPARa inhibition overcomes tumor-derived exosomal lipidinduced dendritic cell dysfunction. Cell Rep. 33, 108278 (2020).

109. Baek, A. E., Yu, Y.-R. A., He, S., Wardell, S. E., Chang, C.-Y. \& Kwon, S. et al. The cholesterol metabolite 27 hydroxycholesterol facilitates breast cancer metastasis through its actions on immune cells. Nat. Commun. 8, 864 (2017)

110. Roberg-Larsen, $H$. et al. Mass spectrometric detection of 27hydroxycholesterol in breast cancer exosomes. J. Steroid Biochem. Mol. Biol. 169, 22-28 (2017)

111. Bahcecioglu, G., Basara, G., Ellis, B. W., Ren, X. \& Zorlutuna, P. Breast cancer models: engineering the tumor microenvironment. Acta Biomater. 106, 1-21 (2020).

112. Greening, D. W., Gopal, S. K., Xu, R., Simpson, R. J. \& Chen, W. Exosomes and their roles in immune regulation and cancer. Semin. Cell Dev. Biol. 40, 72-81 (2015).
113. Myint, P. K., Park, E. J., Gaowa, A., Kawamoto, E. \& Shimaoka, M. Targeted remodeling of breast cancer and immune cell homing niches by exosomal integrins. Diagn. Pathol. 15, 38 (2020).

114. Hoshino, A. et al. Tumour exosome integrins determine organotropic metastasis. Nature 527, 329-335 (2015).

115. Wen, S. W. et al. The biodistribution and immune suppressive effects of breast cancer-derived exosomes. Cancer Res. 76, 6816-6827 (2016).

116. Jiang, $M$. et al. Cancer exosome-derived miR-9 and miR-181a promote the development of early-stage MDSCs via interfering with SOCS3 and PIAS3 respectively in breast cancer. Oncogene 39, 4681-4694 (2020).

117. Taghikhani, A., Hassan, Z. M., Ebrahimi, M. \& Moazzeni, S. M. microRNA modified tumor-derived exosomes as novel tools for maturation of dendritic cells. J. Cell. Physiol. 234, 9417-9427 (2019).

118. Chatterjee, S. et al. Transforming growth factor beta orchestrates PD-L1 enrichment in tumor-derived exosomes and mediates CD8 T-cell dysfunction regulating early phosphorylation of TCR signalome in breast cancer. Carcinogenesis 42, 38-47 (2021). 